\title{
Software Programming with Lumbar Spine Cadaveric Specimens for Computational Biomedical Applications
}

\author{
Francisco Casesnoves"1 \\ "PhD Engineering, MSc Physics, MD. Independent Researcher Engineering-Physics-Software and Medical \\ Bioengineering Devices. IAAM (International Association of Advanced Materials). COE Tallinn University of \\ Technology, Tallinn, Harjumaa, Estonia
}

\begin{abstract}
Article Info
Volume 7, Issue 1

Page Number: 07-15

Publication Issue :
\end{abstract}

January-February-2021

\section{Article History}

Accepted : 01 Jan 2021

Published : 04 Jan 2021

\section{ABSTRACT}

This contribution deals with demonstration of software-computational methods (CAD) to digitalize, simulate, and fit mathematically the anterior vertebral body morphometry of cadaveric lumbar spines. From previous research publications, computational techniques are developed and explained. Based on anatomical-cadaveric spinal specimens, experimental data was implemented to obtain practical surgical applications. With these anatomical samples, large sets of surface digital points were generated. Complete anterior vertebral body morphologies were visualized and analyzed with designed software-engineering. Selection method for extraction of Regions of Interest (ROIs) is presented. Applications of Lumbar anterior, vertebras, and intervertebral disks surfaces, are explained. Further biomedical anatomical analysis applications are included. Results comprise realistic anatomical representations of lumbar spine. Important applications in computational design, surgical robotics, and optimization of surgery tools and techniques are presented.

Keywords : Software Engineering, Surfactal Programming, CAD (Computer Aided Design), CAM (Computer Aided Manufacturing), ROI, Anatomical Cadaveric Simulations, Biomechanics, Bioengineering, Spinal ComputationalSurgery, Surgery Robotics Integrated Systems

\section{INTRODUCTION}

Surgery techniques and methods have evoluted significantly in recent times. Currently, computational techniques, CAD, CAM, are used for design of optimal surgery tools, operation preparation, post-surgery control, and surgical theatre equipment. Computational Surgery field, both for investigation and clinical practice, has emerged as a standard specialization in modern hospitals. Complementary, Robotics Surgery branch constitutes an additional super-specialization to optimize the precision, efficiency and efficacy in particular surgical tasks.

Minimal invasive spinal surgery requires a profound knowledge of the operational field. This can be done with CAD simulations of spine and surrounding biomechanical-related tissues. 
Therefore, the pre-operative planning and preoperative simulations obtain important advantages, precision, and efficiency with these methods. CAD can be also used for spinal surgery training provided it is based on real cadaveric specimens images/surfaces. In addition, CAM in medical industry gets advances and functionality with these realistic developments.

In brief, this contribution shows computational methods for 3D image setting in biomedical CAD and CAM. In particular for lumbar spine with computational surgery applications. Projections towards future applications of surgical robotics are overviewed. Direct applications in spinal surgery are detailed.

\section{ANATOMICAL CADAVERIC MATERIALS AND METHODS}

The CAD surfactal fit was obtained from anterior vertebral body anatomic cadaveric samples $[5,6]$. The cloud data of these cadaveric samples was obtained using the 3D Scanner Digitizer (high resolution $\simeq 10$ ${ }^{7} \mathrm{~m}$ ). The data obtained of the spine surfaces had $\simeq 10^{5}$ points for each specimen. The initial point number is reduced from $\simeq 10^{5}$ to $\simeq 10^{4}$ to increase the visualization program speed. For the cadaveric specimen of this study, number of surfactal points are approximately 1300 .

In previous contributions, extent developments of the specimens for computational programs were carried out $[5,6]$. However, in this paper the focus is on a different cadaveric sample. The study is centered on the programming design, new anatomical information that can be obtained from the anterior lumbar spine, and modern biomedical applications.

\section{SOFTWARE PROGRAMMING TECHNIQUES}

The programming method was based on both subroutines and a group of codes to set spinal images accurately. It was rather complicated the setting of around 2000 cloud points and at the same time to obtain a running time short. However, this difficulty was sorted mainly with two methods. Programming techniques are based on previous publications, $[2,4,5,6,8,9]$. First, selecting the fastest subroutines for 3D imaging. Second, getting an optimal order for the program sentences to avoid unnecessary operations, loops, and reiterations of calculus.

All in all, the average running time for a cadaveric image was 2-4 seconds. The image tools time to start to work was about 2 seconds. The division of numerical intervals for tiles was selected about 0.5 for optimal visualization and not long running time.

\section{RESULTS AND BIOMECHANICAL- ANATOMICAL-IMAGING STUDY}

Computational results are shown in series of imaging surfactal plots, $[2,4,5,6,8,9]$. Images start from the visualization of basic sides of the specimen. Then, step by step, the details are explained. In Figure a cranial view is shown. Figure 2 is a caudal view. In Figure 3 differences between curvature of vertebral surface and disk surface are detailed. The Anterior longitudinal ligament traces are included in Figure 4. Anterior longitudinal ligament creates a different points distribution, because the ligament surface is not smooth, it is ligament fibers. This causes different physical cloud points in scanning process, which is reflected in the image model. In Appendix I, Figs 1 and 2, enhanced views are shown. In Appendix I, Fig 3 , a previous contribution with another different specimen and special software for separation of vertebras and disks is presented. 


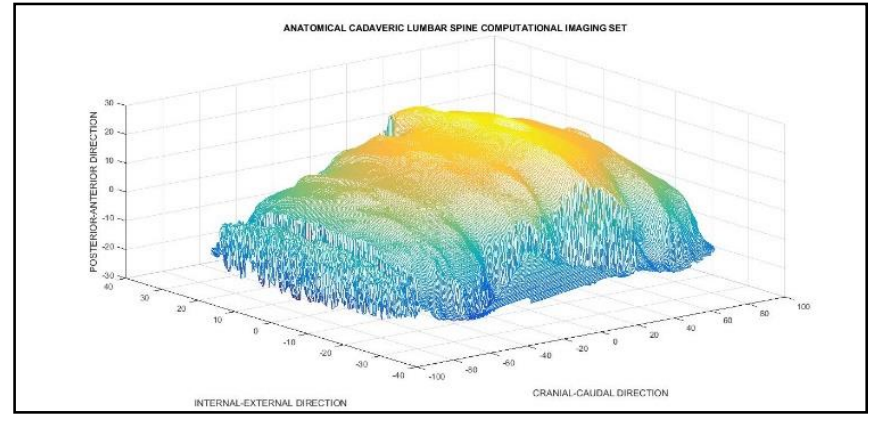

Figure 1.-First cadaveric reconstruction of lumbar spine specimen. Anatomical directions are set. From this CAD image, further analysis of vertebras, disks and ligaments can be done.

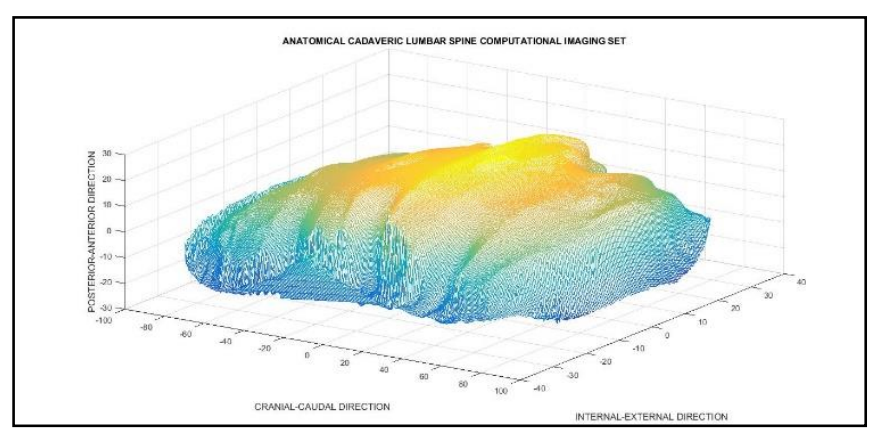

Figure 2.-Caudal view of the lumbar spine specimen. It is shown L4, L5 and part of S1. Disks L4-L5, and L5-S1 can be identified. Anatomical directions are set.

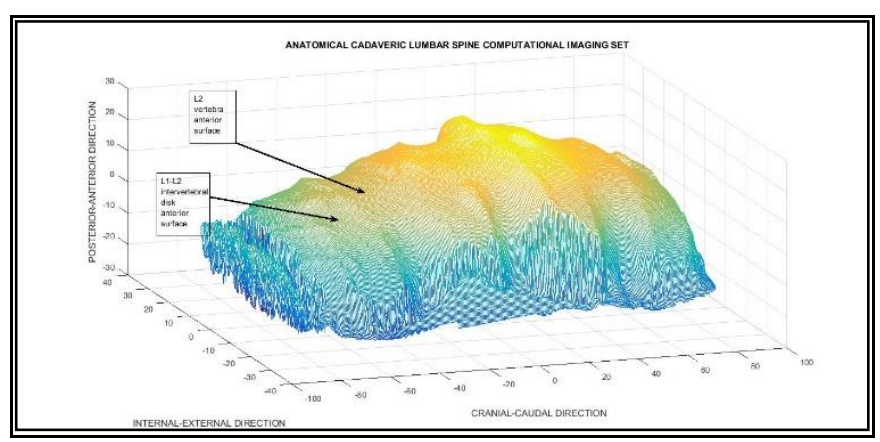

Figure 3.-Cranial view of the lumbar spine specimen. It is shown L2, L3 and disk L2-L3, in legends.

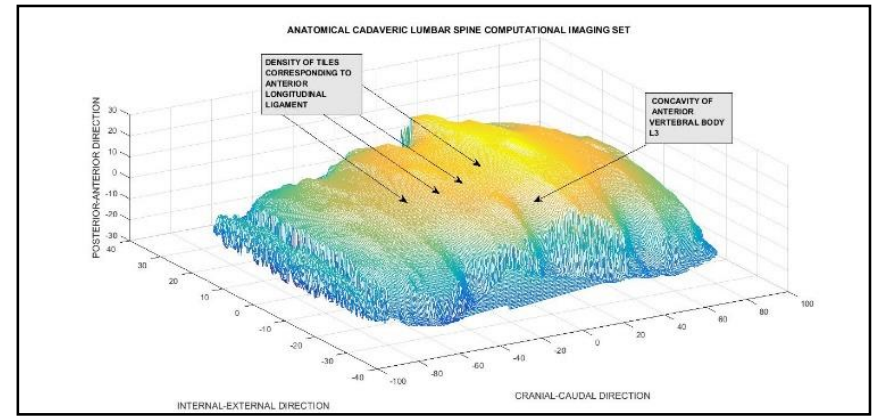

Figure 4.-Cranial view of the lumbar spine specimen. It is shown the different density of tiles corresponding to anterior longitudinal ligament [ALL]. The curvature of the vertebras is more concave than the disks, as it is indicated. Concavity of vertebral bodies is higher than disks for a number of anatomical-physiological and biomechanical reasons. Among them, the disks experience continuous biomechanical pressure and deformations for lumbar loads. Other reason is that these cadaveric specimens correspond to aged corpses, therefore the compression history of the disks is longer. [Appendix 1, Fig. 1].

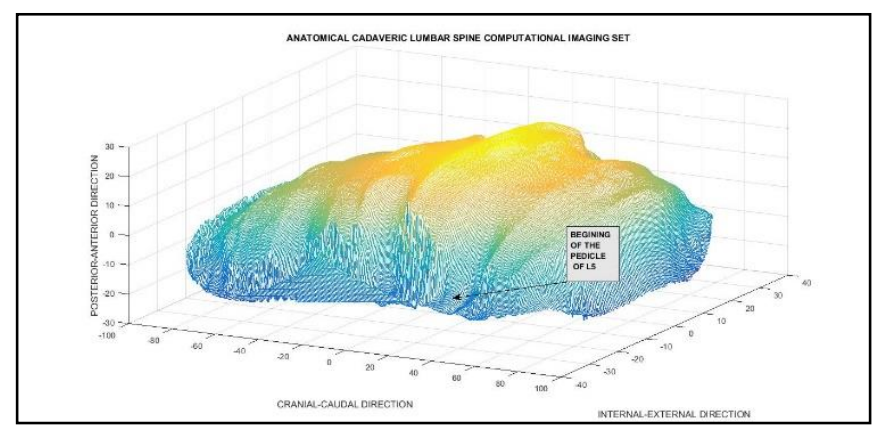

Figure 5.-Caudal view of the lumbar spine specimen. It is shown the begining of the L5 right-pedicle. The scanner took low number of points from the lateral parts of the anterior spine surface. Therefore, it is rather difficult to identify pedicles. There is image of a L5 pedicle also at left side. Anatomical directions are set. 


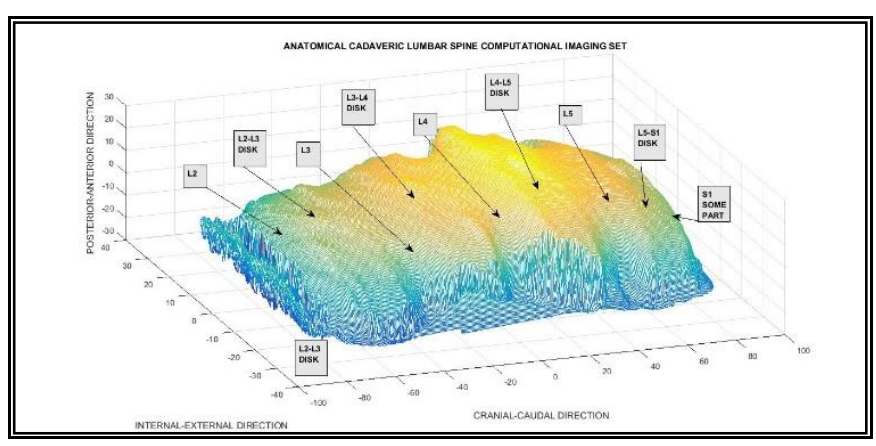

Figure 6.-Complete details of lumbar vertebras and disks distribution in specimen. L2 is scarcely seen, as it happens with S1 in this view. Enhanced in Appendix I, Fig 2.

\section{COMPUTATIONAL-SURGERY AND BIOMECHANICAL PPLICATIONS}

In modern surgery, CAD constitutes an useful method for design of manufactured tools, apparatus, prostheses, and related industrial-medical products. The adaptation/fit among this series of instruments on the spine bone-surface has to be as much precise as possible. The more precision, the better task at the surgical theatre, the optimal saving time in operations, efficacy of the instrumentation, efficiency of the surgical team work, post-operational results, and patient health restoration/cure $[5,6,9]$.

In spinal surgery, both the instrumentation and a large variety of implants have to contact, or be permanently attached to the vertebral bone structure. The functional objective of these surgical tools or unchangeable spinal implants is to exert forces that modify the mechanics and dynamics of the spine, either temporarily in the operating theater or permanently. It is a common mechanical technique to mesh the surgical tools or spinal prostheses with special tools and/or screws [5]. Screws are considered effective in cervical spine surgery, but not in general for the lumbar spine (e. g., Spondylolisthesis surgery) $[5,6]$. This is caused by the different magnitudes of the biomechanical forces corresponding to each spine level. Broadly defined, there are two principal types of spinal screws, according to the vertebral bone structure for which they are designed. The cancellous bone screws reach the interior of the vertebra, while the cortical screw is anchored at the external bone histological layer of the vertebral body. Histocompatibility is crucial for the screws manufacturing, especially in permanent implants. In addition, pedicular screws are extensively used (e.g., in Scoliosis surgery, which is a highly prevalent disease) [5]. Recently, the so-called Bioabsorbable screws (even bioabsorbable spinal cages,), with polymer or composite structure, are being used in spinal surgery, e.g., in transpedicular anatomical zones. Clinical statistical studies concerning proof of complete reabsorption and substitution by osteum tissue have been carried out, although the complete reabsorption and substitution has not been definitely proven $[5,9]$. In general, the same criteria about the bioengineering precision remains for Bioscrews, and this type of screw could provide future, promising technical advantages.

In addition, the design of surgical tools for pediatric surgery is even more complicated sometimes than adult spinal surgery. The intervals of growing-up for the body constitution in children is rather difficult. The spine in children has neither the same adult shape, nor totally equal vertebral bone and/or ligaments hardness/vicoelasticity compared to adults. Computational Biomechanics design constitutes an useful tool to overcome these biomedical difficulties.

\section{BRIEF OF SURGICAL ROBOTICS AND BIOMEDICAL CYBERNETICS APPLICATIONS}

Some statistics have shown that $20 \%$ of surgical robots are used in orthopedics surgery [13]. Advantages for bone and hard tissues performed by robots are the better conditions for exerting precise mechanical forces. Therefore, these hard tissues constitute a good field of work-task for robotic 
integrated systems. Further advantages in orthopedics-robotics, are the better RX, CT, MRI imaging contrast, and resistance to radiation. Additionally, surgical infection prevalence/incidence could have a lower rate.

The future of traumatologic surgical robotics, given these advantages, offers several promising-practical possibilities [Casesnoves, 2019]. Artificial intelligence can be incorporated into the robot integrated system [Casesnoves, 2019]. Forensic Robotics, [Casesnoves, 2019], can be performed with additional good advantages. These are, for instance, that monitoring or anesthesia are unnecessary, the absence of risks for patient life if failure happens, and the time schedule without constraints.

Spinal simulations are therefore useful for Minimal Invasive Robotic Surgery, Pre-operative Spinal Planning, and Simulation of Pre-operative Spinal Surgery.

New studies about the pedicular screw robotic placement show a slight reduction in failure rate with this technique, and a clearly lower relative screw angular deviation. Additionally, when using this robotically guided pedicular insertion [5], the X-Ray average Exposure and surgery duration is also reduced significantly. To complete this futureprospective robotic integrated systems section, just remark that surgical robotics field for veterinary surgical pathology constitute an useful area for investigation and future applications [Casesnoves, 2020].

\section{DISCUSSION AND CONCLUSIONS}

This contribution has shown the programming method to obtain accurate surfactal images from scanning cloud data of cadaveric specimens of lumbar spine. The real anatomical model of the vertebral, anterior longitudinal ligament, some pedicles and disks can be considered acceptable. The software used with subroutines complemented with a specific program has resulted in sharp visualization. However, lateral parts of the spine had not sufficient cloud data to be drafted correctly.

Anatomical analysis of cadaveric specimens was detailed. Specially for surfaces, geometrical characteristics, and complementary soft tissuesnamely, anterior longitudinal ligaments.

Applications of curvatures, concavities geometrical data, angles of curvature, size of surfaces, and deformation of vertebras with ageing specimens are useful for surgical industry. These purposes are suitable for precise surgical tools manufacturing, spinal artificial implants/prostheses,orthopaedic apparatus/instruments, surgical robotics and computational preparation/design of intervention $[1,13]$. In addition, following-up of operation results could be complemented with real cadaveric specimen images. A few of Forensic Robotics engineering concepts, [Casesnoves, 2020], were presented in Surgical Cybernetics, Section VI.

In summary, this paper has demonstrated how imaging information can be obtained from specimens in order to be used with biomedical applications and in surgical-medical industry.

\section{SCIENTIFIC ETHICS STANDARDS}

This contribution is based on Graphical Visualization-Optimization methods for cadaveric specimens of lumbar spine. Graphical-Optimization Methods were created by Francisco Casesnoves on $3^{\text {rd }}$ November 2018, while he was preparing his Doctoral Thesis Defence and publishing some papers in IMRT Radiation Therapy. Forensic Robotics Integrated Systems engineering-concepts were developed by Casesnoves in July 2020. This article has a few previous paper information and figures, whose 
inclusion is essential to make the contribution understandable. This study was carried out, and their contents are done according to the European Union Technology and Science Ethics. Reference, 'European Textbook on Ethics in Research'. European Commission, Directorate-General for Research. Unit L3. Governance and Ethics. European Research Area. Science and Society. EUR 24452 EN [14,15]. This research was completely done by the author, the software, calculations, images, mathematical propositions and statements, reference citations, and text is original for the authors. When anything is taken from a source, it is adequately recognized. Ideas from previous publications were emphasized due to a clarification aim, $[14,15]$.

\section{REFERENCES}

[1] Panjabi, M, White, A. Clinical Biomechanics of the Spine. Lippincott. 1980.

[2] Casesnoves, F. The Numerical Reuleaux Method, a computational and dynamical base with applications. First Part. Lambert Academic Publishing. ISBN-10 3659917478. 2019.

[3] Casesnoves F, Suzenkov A. Mathematical Models in Biotribology with 2D-3D Erosion Integral-Differential Model and Computational-Optimization/Simulation Programming. International Journal of Scientific Research in Computer Science, Engineering and Information Technology. 2017 IJSRCSEIT | Volume 2 | Issue 3 | ISSN : 24563307.

[4] Casesnoves F. Mathematical Models and Optimization of Erosion and Corrosion. Taltech University Press. Doctoral Thesis. ISSN 25856898. 2018.

[5] Casesnoves, F. 'Computational Simulations of Vertebral Body for Optimal Instrumentation Design'. ASME Journal of Medical Devices (Research Paper). Author: F Casesnoves
.Journal of Medical Devices. June 2012. Volume 6. Issue

$2 / 021014.11$ pages.http://dx.doi.org/10.1115/1.4006670.

[6] Casesnoves, F. Applied inverse methods for optimal geometrical-mechanical deformation of lumbar artificial disks/implants with Numerical Reuleaux method. 2D comparative simulations and formulation. Ethan Publishing Computer Science Applications. 2015, 2, 4, pp. 1-10. [USA Congress Library registration].

[7] Casesnoves, F. 'A Monte-Carlo Optimization method for the movement analysis of pseudorigid bodies'. 10th SIAM Conference in Geometric Design and Computing, Texas, San Antonio, USA. Contributed Talk. November 2007.

[8] Casesnoves, F. 'Applied Inverse Methods for Deformable Solid Dynamics/Kinematics in Numerical Reuleaux Method (NRM)'. International Journal of Numerical Methods and Applications. volume 9(2) 2013 .pages 109131. peer-reviewed International Mathematical/Computation Journal Article. print/Online.http://www.pphmj.com/abstract/7 688.htm. This article is specially innovative in Inverse Problems applications for deformable solids kinematics/dynamics, further publications are included in United States Congress Library and Numerical Reuleaux Method is accepted by scientific community as an innovative dynamics method in deformable solids with mechanical, biomechanical and aerospace applications. New applications of this method will be probably found significantly in future.

[9] Casesnoves, F. Nonlinear comparative optimization for biomaterials wear in artificial implants technology. Presented in Applied Chemistry and Materials Science RTU2018 Conference Proceedings. 2018. 
[10] Casesnoves, F. Applied Inverse Methods for Optimal Deformation of Lumbar Artificial Disk/Implants with Numerical Reuleaux Method and 3D Voxelization-Computational Simulations. American Institute of Science. Bioscience and Bioengineering. Vol. 1, No. 4, 2015, pp.

94-105. http://www.aiscience.org/journal/bio.

[11] Casesnoves, F. 3D Improved mathematical model for lumbar intervertebral ligaments (LILs). Proceedings SIAM Life Sciences Conference joint to SIAM Annual Conference San Diego. 2012, pp. 25-27.

[12] Todinov, M. Reliability and Risk Models. Wiley. 2005.

[13] Rosen, J, Hannaford, B. Satava, R. Surgical Robotics. Wiley. 2011.

[14] 'European Textbook on Ethics in Research'. European Commission, Directorate-General for Research. Unit L3. Governance and Ethics. European Research Area. Science and Society. EUR 24452 EN.

[15] The European Code of Conduct for Research Integrity. Revised Edition. ALLEA. 2017.

\section{AUTHOR'S BIOGRAPHY}

Francisco Casesnoves is Engineering and Natural Sciences $\mathrm{PhD}$ by Talllinn University of Technology (started thesis in 2016, thesis defence/PhD earned Defence in December 2018, official graduate Diploma 2019), Estonia, and computationalengineering/physics independent researcher at COE, MSc-BSc, Physics/Applied-Mathematics (Public Eastern-Finland-University), Graduate-with-MPhil, in Medicine and Surgery (Public Madrid University Medicine School). Casesnoves studied always in public-educational institutions. His education/scientific vocation was motivated very young, by Profs C Navamuel and I Vela, in Renaissance-Humanism ideas-later on with the motivation manuscripts of Nobel and Von Helmholtz prizes Santiago Ramon y Cajal. His constant service to International Scientific Community and Estonian technological progress (2016-present) commenced in 1985 with publications in Medical Physics, with further specialization in optimization methods in 1997 at Finland — at the moment approximately 100 recognized publications with 50 papers. His main branch is Computational-mathematical Nonlinear/Inverse Methods Optimization. Casesnoves best-achievement is the Numerical Reuleaux Method in dynamics and nonlinearoptimization [books 2019-2020]. Casesnoves scientific service since 2016 to the Free and Independent Republic of Estonia for technological development (and also at Riga technical University, Power Electrical and Electronics Department) is about 25 physics-engineering articles, two books, and 1 industrial radiotherapy project associated to Europe Union EIT Health Program (Tartu University, 2017).

\section{Cite this article as :}

Francisco Casesnoves, "Software Programming with Lumbar Spine Cadaveric Specimens for Computational Biomedical Applications", International Journal of Scientific Research in Computer Science, Engineering and Information Technology (IJSRCSEIT), ISSN : 2456-3307, Volume 7 Issue 1, pp. 07-13, January-February 2021. Available at

doi : https://doi.org/10.32628/CSEIT206663

Journal URL : http://ijsrcseit.com/CSEIT206663 


\section{APPENDICES}

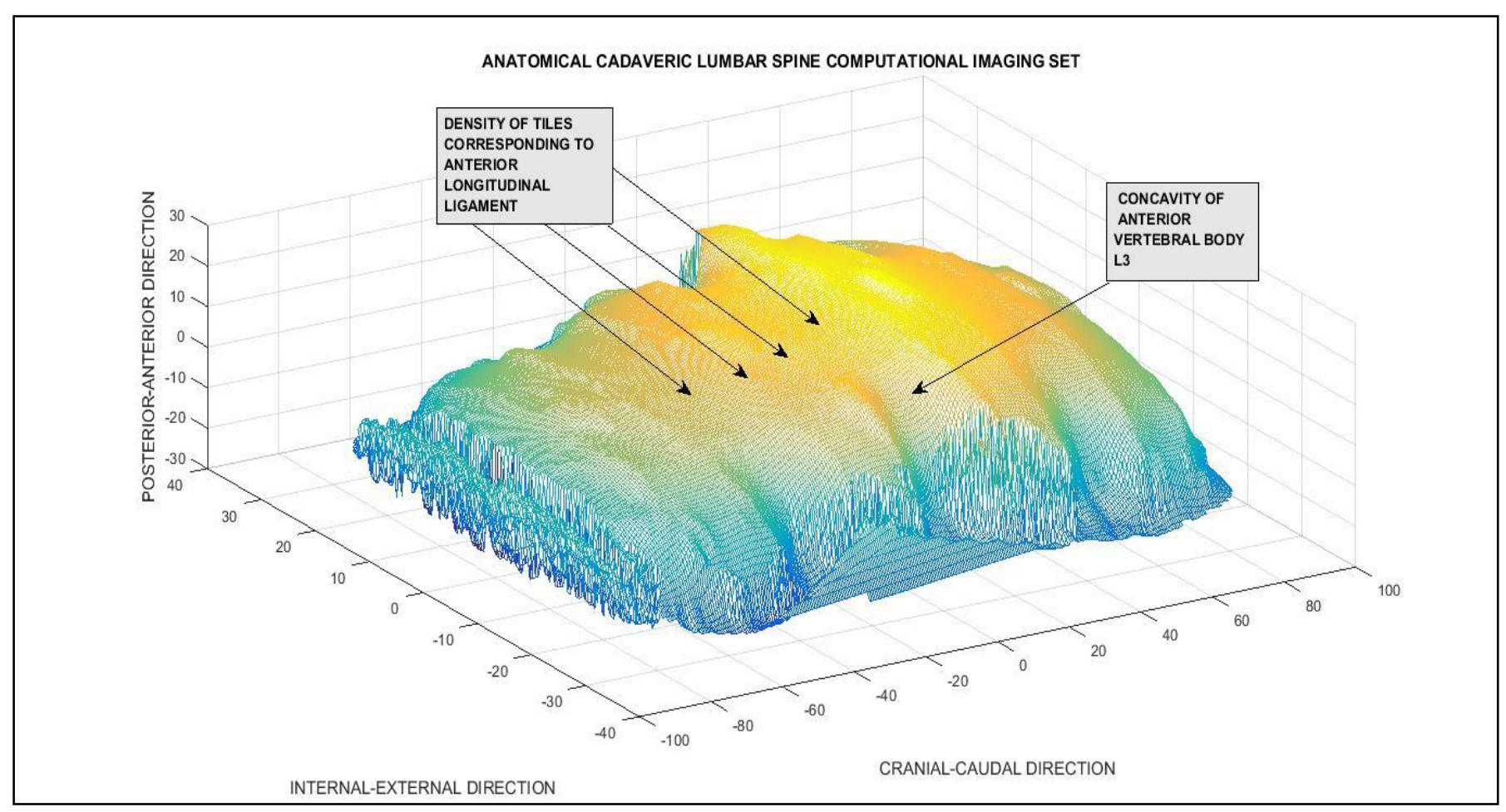

Figure 1.-Cranial view showing traces of anterior longitudinal ligament and L4 vertebral body surface [erratum, in legend should be L4, not L3].

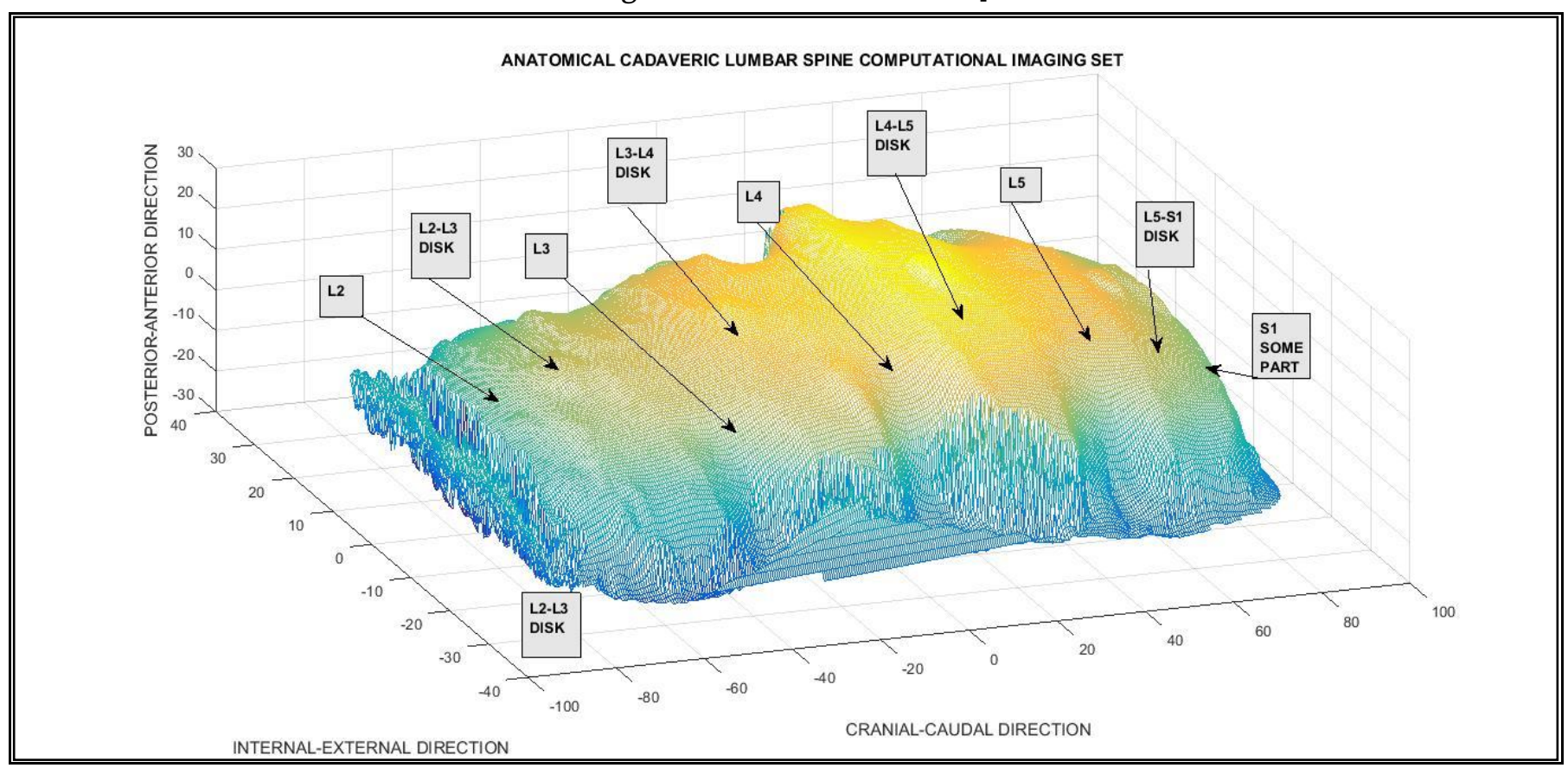

Figure 2.-Complete enhanced details of cadaveric sample. 


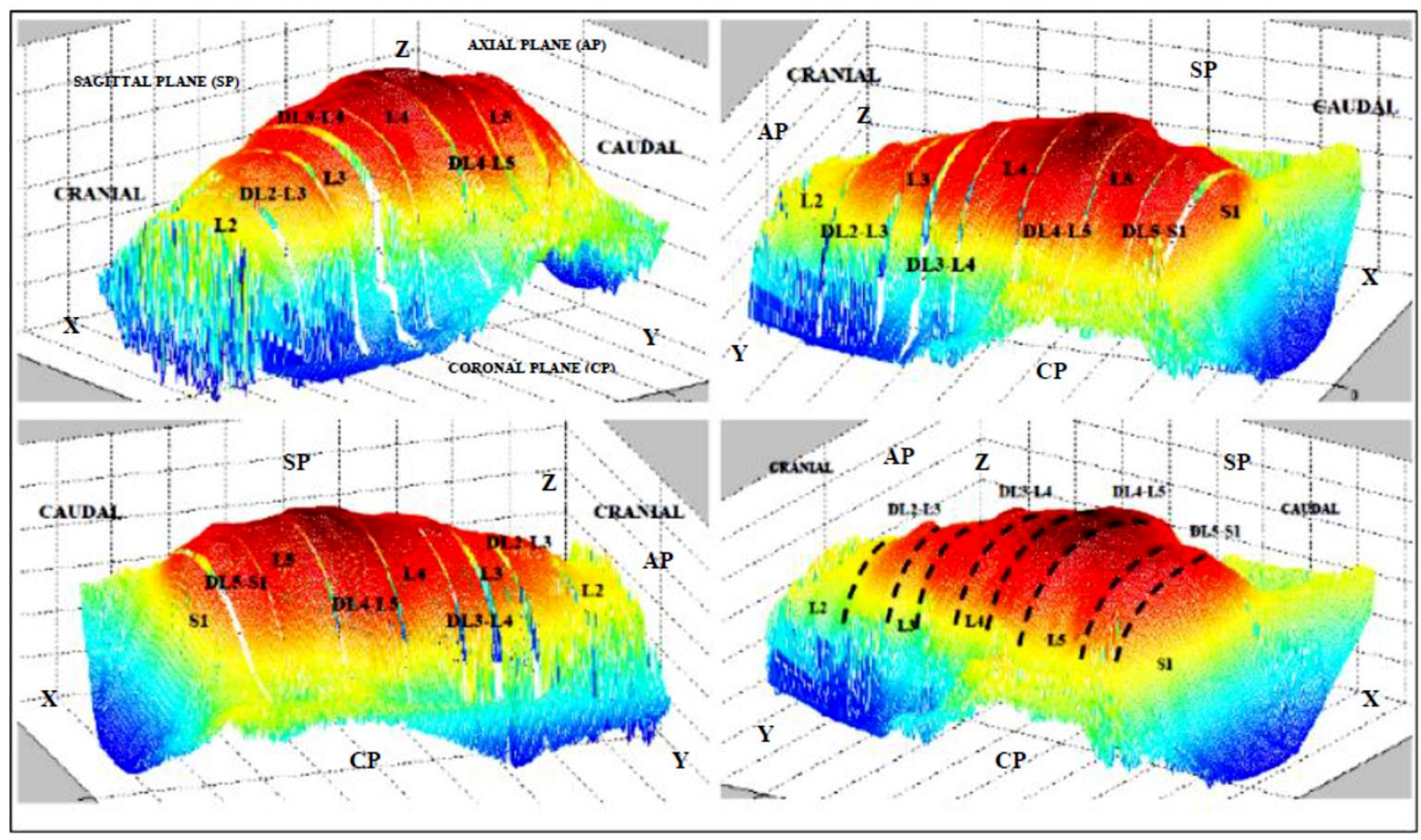

Figure 3.-Previous contribution with special software to show separated vertebras and disks of a different anatomical specimen [5]. 